RUNNING HEAD: COLOR OF A NATION

Orange as a Perceptual Representation of the Dutch Nation: Effects on Perceived National Identification and Color Evaluation

\author{
Daniël Lakens \\ Eindhoven University of Technology \\ In Press, European Journal of Social Psychology
}

Words: 4631

Correspondence should be addressed to Daniël Lakens, Human Technology Interaction

Group, IPO 1.24, PO Box 513, 5600MB Eindhoven, The Netherlands. E-mail:

D.Lakens@tue.nl. 


\begin{abstract}
Although it is generally accepted that colors carry meaning, experimental research about individual, situational and cultural differences in the meaning of colors is scarce. The current research examines whether the Dutch national color functions as a perceptual representation of The Netherlands. A person dressed in orange clothing was judged to identify more with his nation compared to the same person dressed in blue (Study 1). When national identification was salient, such as during (vs. before/after) the European soccer championship, or when participants recalled an experience in which they identified (vs. not-identified) with The Netherlands, and people were more aware of the use of the color orange as a perceptual representation of The Netherlands, orange was evaluated more positively (Studies 2 and 3). Furthermore, orange evaluations correlated with self-reported national identification. These results support the hypothesis that national colors carry psychological meaning, which can influence person perception and color evaluations.
\end{abstract}

Keywords: color, orange, national identification, perceptual representation, evaluation. 


\section{Orange as a Perceptual Representation of the Dutch Nation: Effects on Perceived National Identification and Color Evaluation}

The meaning of colors is a hugely popular topic in folk psychology. People take time to choose the best color tie to wear to a job interview, and companies carefully weigh their options when choosing a color for their logo. This general interest in the meaning of colors is not reflected in psychological science. Although the idea that colors carry meaning is generally accepted, the scientific investigation of the affective and behavioral consequences of color associations has only recently gained prominence. The meaning of colors is grounded in two sources (Elliot, Maier, Moller, Friedman, \& Meinhardt, 2007; Elliot \& Maier, 2007). On the one hand, the literature suggests that colors carry evolutionary predisposed meanings. For example, red is associated with avoidance (Elliot et al., 2007) and romance (Elliot \& Niesta, 2008), and black is associated with negativity (Adams \& Osgood, 1973). On the other hand, colors can acquire meaning through learned associations by repeatedly being paired with specific concepts. Such color associations can become so strong that merely perceiving a color unconsciously activates its associated meaning, subsequently influencing affect, cognition, or behavior (Elliot et al., 2007). Despite the ubiquity of color information in daily life, relatively little is known about how color meanings differ across cultures, situations, and individuals. This article focuses on culturally learned associations between a nation and the national color.

The color of the national flag is often used as a visual representation of a nation. Flags are symbolic conceptual representations of group membership that can activate associations with their nation (Butz, Plant, \& Doerr, 2007; Ferguson \& Hassin, 2007; for a review, see Butz, 2009). In addition to symbolic representations of nations such as flags, buildings, or other cultural icons (e.g., the Capitol or Mickey Mouse as symbols for America and the Summer Palace and a dragon as a symbol for China, see Hong, Morris, Chiu, \& Benet- 
Martínez, 2000), many countries have national colors that are used as perceptual representations of the nation. For example, sport teams dress in the colors of their nation during international competitions to indicate their country of origin.

In the current research, the hypothesis is tested that through the repeated use of the national color as a perceptual representation of a nation, the national color has acquired a psychologically meaningful association with the nation of individuals. Some preliminary research findings already suggest that people evaluate their national color more positively than the national colors of other countries. Burkitt, Tala, and Low (2007) report that Finnish children were less likely to use blue and white colors (the colors of the Finnish flag) to draw a picture of a nasty man than English children. Choungourian (1968) observed that blue and red (the colors of the American flag) were liked by American students and disliked by Kuwaiti students, whereas the reverse was true for a blue-green color (the color of the Kuwaiti flag). Although these findings provide a first indication that the national color is liked more because it is seen as a representation of the nation, these studies did not measure underlying psychological constructs such as national identification. Furthermore, differences in color evaluations between countries can be due to cultural differences unrelated to the national color. For example, green is a special color in Islam (Gage, 1999).

The current research aims to provide more conclusive support for the assumption that color evaluations and national identification are related because the national color functions as a perceptual representation of one's nation. If so, the association between the national color and the nation it represents should manifest itself in two ways. First, seeing the national color should activate information related to one's nation. For example, irrelevant perceptual cues such as clothing items in the national color might bias observers to perceive a person as being more nationalistic. Second, depending on whether the relationship between the nation and its national color are salient or not, evaluations of the national color should become more or less 
positive. Given that people generally identify with their nation (e.g., Leach, Spears, Branscombe, \& Doosje, 2003), and are on average positive about their ingroup (Brewer, 2007), the national color should be evaluated more positively in situations when it is salient that the color is a perceptual representation of the nation, compared to when this relationship is less salient.

\section{Overview of the Studies}

The studies reported below were performed in The Netherlands, and therefore the research question focuses on the Dutch national color, orange ${ }^{1}$. At international sport events, Dutch teams wear orange uniforms, and a frequently used pars pro toto to refer to the Dutch national soccer team is 'Oranje' (Orange). Study 1 investigates the possibility that if the color orange functions as a perceptual representation of The Netherlands, a person wearing orange clothing might be rated higher on perceived national identification for dressing in a color that represents his or her nation than the same person wearing clothing in a different color. The possibility that color associations influence person perception is examined in Study 1. The subsequent two studies investigate evaluations of the national color under circumstances when identifying with The Netherlands is salient for Dutch participants, compared to when it is not. Orange evaluations were investigated during the European soccer championship and compared to orange evaluations before and after the European Championship (Study 2). Study 3 experimentally manipulated the salience of national identification and the association between orange and the Dutch national color by asking participants to recall an experience in which they identified with The Netherlands or not.

\section{Study 1}

Previous studies have revealed that clothing color can influence judgments about an unknown person. For example, Elliot and Niesta (2008) presented men with pictures of woman, and asked them to indicate how sexually attractive the women were. In a series of 
studies, men rated women who wore red clothes as more attractive than women wearing green, white or grey. The current study used a similar manipulation to investigate whether clothing color would influence perceived levels of national identification. A person wearing orange clothing should be judged to identify more strongly with The Netherlands than a person wearing clothing of a different color.

\section{Method}

Participants. Fifty-six students (34 male, mean age 23.48) participated in the current study and were randomly assigned to the orange vs. light blue T-shirt condition.

Procedure. As a cover story, participants were told they would participate in a study in which first impressions and memory were investigated. It was told that some participants would only briefly see a picture of a person, before answering questions about that person, whereas other participants would answer the questions while the picture of the individual remained on the screen. In reality, the latter procedure was used for all participants.

Participants then viewed a picture of a man who was dressed in jeans and either an orange Tshirt (average RGB: 246, 74, 34) or a light-blue T-shirt (average RGB: 141, 192, 224). The color of the T-shirt was digitally altered in a photo-editing program by a clothing company selling T-shirts of different colors. A blue color was chosen because pilot testing revealed that orange and light-blue were evaluated equally positive (on a scale from 1 to $9, M=6,83$ and $M$ $=7,00$, respectively, $t(69)=0.77, p=.44$ ). After 15 control questions (e.g., how old, smart, attractive, well-dressed, unfriendly, creative, dominant, etc. is the person on the picture), participants filled out six items of the perceived national identification scale, based on items used to measure national identification in previous research (e.g., 'The fact that the person in the picture is Dutch is an important part of his identity', 'The person on the picture thinks the Dutch have a lot to be proud of', 'The person on the picture is happy to be Dutch', see Leach, et al., 2003). Responses were given on a 7-point Likert-type scale that ranged from 1 (I very 
much disagree) to 7 (I very much agree). The same measure of perceived national identification was used in all three studies.

\section{Results and Discussion}

The 6 national identification questions were averaged. The reliability was acceptable $(\alpha=.61)$, although slightly lower than in Study $2(\alpha=.87)$. The person in the orange T-shirt was judged to identify more strongly with the Netherlands $(M=4.57, S D=0.65)$ than the person in the light-blue T-shirt $(M=4.15, S D=0.71), t(54)=2.34, p=.023$. Of the 15 filler items, only one question differed between the orange and blue T-shirt conditions (judgments of how young the person was, $M=2.10, S D=0.85$ and $M=2.65, S D=0.89$, respectively, $t(54)=2.38, p=.02$, but controlling for perceived age when comparing ratings of perceived national identification did not influence significance levels, $p=.028)$. The gender of the participants did not influence the results.

These results show that a person who dresses in a color that represents the nation is rated higher on national identification than a person dressed in an equally positive color that does not represent the nation. The relationship between orange and The Netherlands can influence person perception.

\section{Study 2}

Study 1 provides initial support for hypothesis that orange is a perceptual representation of The Netherlands by showing that orange clothing influences the extent to which a person is judged to identify with his nation. The current study examines the effect of individual and situational differences in the salience of national identification on evaluations of the national color. The first hypothesis is that during times when national identification is salient, people are more aware of the use of orange as the Dutch national color, compared to times when national identification is less salient. Because the Dutch on average hold positive attitudes towards The Netherlands (Leach et al., 2003), greater awareness of the fact that 
orange is a perceptual representation of the nation should increase the positivity of orange evaluations. The European soccer championship is an event during which national identification is extremely salient in The Netherlands, and when people are constantly reminded of fact that orange is the national color. To investigate situational differences in the salience of orange as the national color, the data reported in the current study data were collected in three different weeks: Several months before the European soccer championship in 2008, during the European soccer championship, and several months after the European soccer championships was over. The second prediction of the current investigation is that individual differences in the extent to which Dutch participants identify with The Netherlands will influence evaluations of the national color. The more strongly people identify with The Netherlands, the more positive their evaluations of the color orange are expected to be.

\section{Method}

Participants. A total of 218 Dutch students $\left(152\right.$ females, mean age $\left.=20.90^{2}\right)$ participated in only one of the three measurement times. All participants were students at a Dutch university, and were recruited through the same procedure. A chi-square test did not reveal significant differences in the number of males and females over the three measurement times. Data was collected before $(n=34)$, during $(n=70)$ or after $(n=114)$ the European soccer championship in 2008.

Procedure. Data was collected in 3 separate weeks: the pre-measurement was performed from the $22^{\text {nd }}$ to the $26^{\text {th }}$ of October 2007, the data collection during the European soccer championship was collected from the $16^{\text {th }}$ to $20^{\text {th }}$ of June, 2008, with the postmeasurement being collected from the $27^{\text {th }}$ to the $31^{\text {st }}$ of October 2008. The Dutch national soccer team won their first match against Italy on the $9^{\text {th }}$ of June, and won their second match against France on the $13^{\text {th }}$ of June. On the $17^{\text {th }}$ of June the Dutch team won against the Romanian team, and was the best performing team in the European championship ${ }^{3}$. The color 
evaluations and national identification questionnaires were added to other experiments, with the unforeseen consequence that sample sizes were not balanced between measurement times.

Participants were asked to evaluate the color orange (the Dutch national color), and the colors red, white, blue, black, green and yellow ${ }^{4}$ in fixed order on a scale from 1 (very negative) to 9 (very positive). A bright orange was used (RGB: 254, 137, 67) which closely resembles the orange of the Dutch national soccer team, and other Dutch national institutions, such as the state lottery. After an unrelated experiment, the national identification questionnaire was presented as a separate study. After answering all questions, participants were thanked, paid and debriefed.

\section{Results and Discussion}

A repeated measures ANOVA on the seven color evaluations with measurement time as between participants factor revealed the predicted interaction between color evaluation and measurement time, $F(8.93,959.86)=2.93, p=.002$ (Greenhouse-Geisser corrections were applied because the sphericity assumption was violated). Evaluations differed significantly between colors, $F(4.46,959.86)=52.93, p<.001$, but there was no main effect of measurement time, $F(2,215)=1.51, p=.22$.

The evaluations of the color orange were entered in a regressions analysis with the three measurement times (coded as two dummy variables with $0=$ the measurement during the European championship and the 1 = the pre- or post-measurement), mean-centered national identification and the interactions between measurement time and national identification as predictors. As expected, evaluations of the color orange were predicted both by self-reported national identification as by the measurement time. First, orange was evaluated more positively during the European championship $(M=7.26, S D=1.66)$ compared to before $(M=5.21, S D=2.28)$ and after $(M=6.42, S D=2.15)$ the European championship, $\beta=-.39, \mathrm{p}<.001$ and $\beta=-.23, p=.01$, respectively. Second, national 
identification significantly predicted evaluations of the color orange. People who identified more with The Netherlands reported more liking for the color orange, $\beta=.20, p<.01$. Significant levels were identical when Games-Howell post-hoc comparisons were performed that take into account the differences in sample size between different measurement times. This test indicates that it is unlikely that the size differences of the convenience samples influence the results.

The interactions between national identification and measurement time did not reach significance $(p$ 's $>.75)$, indicating that the relation between national identification and evaluations of the color orange did not differ over measurement times. Performing additional multiple regression analyses with a Bonferroni correction on the remaining six colors did not reveal any effect of measurement time, national identification or their interactions (all $p$ 's > .10). Gender of the participants did not influence color evaluations.

Although one might predict that national identification would increase during the European soccer championship, an analysis of variance of the self-reported national identification with time of measurement as independent variable revealed that national identification actually slightly decreased during the European Championship $(M=4.57, S D=$ 1.09) compared to before $(M=5.15, S D=1.30)$ and after $(M=4.99, S D=1.18), F(2,215)=$ $3.87, p=.02, \mu_{\mathrm{p}}{ }^{2}=.03$. Therefore, the more positive evaluations of the color orange during the European championships are not the result of overall higher levels of national identification, but seem to be due to the increased awareness of orange as a perceptual representation of The Netherlands during times when national identification is salient.

Study 2 reveals that evaluations of the Dutch national color is influenced by situational factors (large international sport events), as well as on individuals' level of national identification. During times when identification with The Netherlands is extremely salient, people are more aware of the use of the color orange as a perceptual representation of The 
Netherlands, which provides one possible explanation for the observation that the average evaluation of the color orange increased substantially during the European soccer championship. The hypothesis that salience of national identification plays a role in the differences in orange evaluations between the measurement times is further supported by the observation that the extent to which individuals reported to identify with The Netherlands influenced their evaluations of the color orange.

The quasi-experimental set-up of Study 2 shows how real-life international events can influence color evaluations, but lacked experimental control. It is possible that other factors than the salience of national identification varied between measurement times, and accounted for the observed differences in evaluations of the color orange. For example, evaluative conditioning processes might have increased the positivity of the color orange by seeing the Dutch soccer team win soccer matches, or associating the color orange with enjoyable social events such as watching the game together with friends. Therefore, an experiment was conducted to conceptually replicate the current findings in a lab setting and provide more conclusive support for the importance of the increased salience of national identification.

\section{Study 3}

The current study aimed to replicate the findings of Study 2 in a more controlled setting by asking participants to recall an experience during which they identified with The Netherlands, or an experience during which they did not identify with The Netherlands. When national identification is salient, people were expected to be more aware of the use of the color orange as a perceptual representation of The Netherlands. Following this rationale, and building on the findings of Study 2, the prediction in the current study was that participants would evaluate the color orange more positively after they had recalled an experience during which they strongly identified with The Netherlands, compared to participants who just recalled an experience during which they did not identify with the Netherlands. 


\section{Method}

Participants. Seventy-seven students (45 men, mean age 28.43) participated in the current study and were randomly assigned to the identify vs. not-identify condition.

Procedure. Participants were told they would participate in several unrelated studies. First, participants were asked to recall a personal experience in which they either identified or did not identify with The Netherlands, and to write down what happened and how they felt at that moment. Immediately after this task, participants indicated their current mood on a horizontal line ranging from very negative to very positive, and subsequently rated 14 colors on their valence (in addition to the 7 colors of Study 2, dark green, brown, purple, pink, gold, grey, and light blue were added).

\section{Results and Discussion}

As expected, participants who had just recalled an experience during which they identified with The Netherlands evaluated the color orange more positively $(M=7.31, S D=$ 1.07) than participants who just recalled an experience during which they did not identify with The Netherlands $(M=6.34, S D=2.14), t(75)=2.57, p=.012$. Participants in the two conditions did not differ in their mood after they wrote down an experience during which they did or did not identify with The Netherlands $(t<1)$, nor were there any differences in evaluations of the other colors in the color evaluation task (all $p$ 's $>.16$ ). Gender of the participants did not influence the results.

When examining the experiences participants recalled, 21 of the 42 participants in the identify condition referred to international sport events. Four participants described a national holiday celebrating the Queen's birthday. During these events, people tend to dress up in orange clothing (which is sometimes referred to as 'Oranjekoorts', or Orange Fever). The remaining 17 participants described other experiences during which they identified with the Netherlands (e.g., coming home after a long trip abroad). In the not identify condition, 
participants described experiences of living abroad, disagreeing with political developments in The Netherlands, or being embarrassed by the behavior of Dutch people. Two participants spontaneously recalled events related to international soccer championships, providing anecdotal evidence for the observation in Study 2 that some Dutch inhabitants identify less with The Netherlands during international soccer events.

The current results provide an experimental replication of the findings of Study 2 . When a personal experience during which people identified with The Netherlands was salient, the color orange was evaluated more positively than when an experience in which participants did not identify with the Netherlands was salient.

\section{General Discussion}

Three studies provide support for the hypothesis that orange, the Dutch national color, functions as a perceptual representation of The Netherlands. A person is judged to identify more strongly with The Netherlands if he wears an orange T-shirt, compared to a blue T-shirt (Study 1). Evaluations of the color orange were higher during the European soccer championship (when national identification is salient and people are more aware of the association between orange and The Netherlands) compared to before and after the championship, and correlated with self-reported national identification. Finally, an experiment revealed that participants who were asked to recall a time when they identified with The Netherlands evaluated the color orange more positively than participants who were asked to recall a time when they did not identify with The Netherlands. The current work adds to emerging literature on color psychology by showing that culturally determined colors associations can influence person perception and color evaluations as a function of individual and situational differences.

Previous work on cultural differences in color evaluations has compared color evaluations between countries (e.g., Burkitt et al., 2007; Choungourian, 1968; Saito, 1996), 
and often provides a post-hoc rationale for observed differences. For example, Adams and Osgood (1973) explain the relatively negative evaluation of the color blue in Greece by the fact that it is the name of the least successful professional soccer team in that country. Other research has provided descriptive overviews of cultural differences in color associations, without experimentally investigating the psychological meaning of colors in these cultures (e.g., Madden, Hewitt, \& Roth, 2000). By investigating the relationship between the psychological construct of national identification and the national color, the current results provide more conclusive support for the assumption that national colors can function as a perceptual representation of the nation. By relating color evaluations to psychological constructs, the current findings go beyond comparisons of color evaluations. This approach provides an important avenue for future research into the meaning of colors. Previous studies have primarily focused on the color red, due to its strong biologically predisposed function as an attention cue (Elliot et al., 2010; Elliot \& Aarts, 2011; Elliot \& Niesta, 2008; Hagemann, Strauss, \& Leissing, 2008; Hill \& Barton, 2005; Khan, Levine, Dobson, \& Kralik, 2011). The current article is one the first to broaden the scope of color psychology by providing empirical support for the psychological meaning of culturally learned color associations.

The finding that the national color can function as a perceptual representation of the nation could have a wide range of applications. Companies can use the increased positivity of orange in times when the salience of orange as perceptual representation of The Netherlands is strong to their advantage, for example by selling orange versions of their products, using orange in their advertisements, or if their company logo is orange, by displaying it more prominently when international sport events are in progress ${ }^{5}$. Using national colors to indicate group membership should be especially efficient for tourists abroad who try to follow their guide during a tour. Consumers should perhaps think twice about buying a new orange car during the European championship, because they might regret the purchase several months 
later. In addition to practical applications of the current findings, future research could investigate whether the current observations generalize to other nations and their national colors.

One might have predicted an interaction between measurement time and national identification in Study 2, with national identification being more predictive of the evaluation of orange during the European Championship compared to before or after. The fact that this was not the case might be explained by the surprising decrease in national identification during the European championship. Although national identification should increase during the championship for soccer fans, people who are not soccer fans might actually identify less with their nation during these times. Future research should benefit from measuring general interest in soccer, and might then reveal a three-way interaction, with evaluations of the color orange depending on participants being a soccer fan or not, national identification, and measurement time. Perhaps national identification is a stronger predictor for evaluations of the color orange for soccer fans, and a weaker predictor for people who dislike soccer, especially during the European championship. The salience of orange as a visual representation of The Netherlands is nevertheless increased for everyone, explaining more positive evaluations for the color orange in general.

Previous research has stressed the importance of controlling for differences in brightness and saturation when performing color research. Controlling for brightness is important because brighter colors are often evaluated more positively than darker colors (Valdez \& Mehrabian, 1994). Although many studies control for brightness, it may be more important to equate the colors in psychological research on psychological characteristics (e.g., valence). In Study 1, the color of the T-shirt in the control condition was intentionally chosen to be equally positive as orange to exclude the possibility that effects could be accounted for by valence differences. Furthermore, 15 control questions were added that would reveal 
differences between conditions if valence or other factors (e.g., dominance) could account for the observed effect on national identification. In Studies 2 and 3, color evaluations are collected for a wide range of up to 14 colors. Some colors have comparable luminance levels, others have comparable saturation levels, but effects are only predicted and observed for the color orange. The goal of this approach is to make sure that any observed difference is most parsimoniously explained by the hypothesis that national identification and the colors orange are conceptually related in Dutch participants.

Colors carry psychological meaning. A better awareness of the way color associations differ between individuals, situations, and cultures is hugely important in our contemporary globalizing society. Given the massive interest for this field outside of science, psychologists should take up the challenge to provide empirical insights into the meaning of colors. 


\section{References}

Adams, F. M., \& Osgood, C. E. (1973). A cross-cultural study of the affective meanings of color. Journal of Cross-Cultural Psychology, 4, 135-156. DOI:

$10.1177 / 002202217300400201$

Berlin, B., \& Kay, P. (1969). Basic color terms: Their universality and evolution. Los Angeles: University of California Press.

Brewer, M. B. (2007). The social psychology of intergroup relations: Social categorization, ingroup bias, and outgroup prejudice. In A. W. Kruglanski \& E. T. Higgins (Eds.), Social psychology: Handbook of basic principles (2nd ed., pp. 695-715). New York: Guilford Press.

Burkitt, E., Tala, K., \& Low, J. (2007). Finnish and English children's color use to depict affectively characterized figures. International Journal of Behavioral Development, 31, 59-64. DOI: 10.1177/0165025407073573

Butz, D. A. (2009). National Symbols as Agents of Psychological and Social Change. Political Psychology, 30, 779-804. DOI: 10.1111/j.1467-9221.2009.00725.x

Butz, D. A., Plant, E. A., \& Doerr, C. E. (2007). Liberty and justice for all? Implications of exposure to the U.S. flag for intergroup relations. Personality and Social Psychology Bulletin, 33, 396-408. DOI: 10.1177/0146167206296299

Choungourian, A. (1968). Color preference and cultural variation. Perceptual and Motor Skills, 26, 1203-1206. DOI: 10.2466/PMS.26.3.1203-1206

Elliot, A. J., \& Aarts, H. (2011). Perception of the color red enhances the force and velocity of motor output. Emotion, 11, 445-449. DOI: 10.1037/a0022599

Elliot, A. J., \& Maier, M. A. (2007). Color and psychological functioning. Current Directions in Psychological Science, 16, 250-254. DOI: 10.1111/j.1467-8721.2007.00514.x 
Elliot, A. J., Maier, M. A., Moller, A. C., Friedman, R., \& Meinhardt, J. (2007). Color and psychological functioning: The effect of red on performance attainment. Journal of Experimental Psychology: General,136, 154-168. DOI: 10.1037/0096-3445.136.1.154

Elliot, A. J., \& Niesta, D. (2008). Romantic red: Red enhances men's attraction to women. Journal of Personality and Social Psychology, 95,1150-1164. DOI: 10.1037/00223514.95.5.1150

Elliot, A. J., Niesta Kayser, D., Greitemeyer, T., Lichtenfeld, S., Gramzow, R. H., \& Maier, M. A. (2010). Red, rank, and romance in women viewing men. Journal of Experimental Psychology: General, 139, 399-417. DOI: 10.1037/a0019689

Ferguson, M. J., \& Hassin, R. R. (2007). On the automatic association between America and aggression for news watchers. Personality and Social Psychology Bulletin, 33, 16321647. DOI: 10.1177/0146167207307493Gage, J. (1999). Color and meaning: Art, science and symbolism. Berkeley, CA: University of California Press.

Hagemann, N., Strauss, W., \& Ließing, J. (2008). When the referee sees red. ... Psychological Science, 19, 769-771. DOI: 10.1111/j.1467-9280.2008.02155.x

Hill, R. A., \& Barton, R. A. (2005). Red enhances human performance in contests. Nature, 435, 293. DOI: $10.1038 / 435293 a$

Hong, Y., Morris, M., Chiu, C. Y., \& Benet-Martínez, V. (2000). Multicultural minds: A dynamic constructivist approach to culture and cognition. American Psychologist, 55, 709-720. DOI: 10.1037//0003-066X.55.7.709

Khan, S. A., Levine, W. J., \& Dobson, S. D., \& Kralik, J. D. (2011). Red signals dominance in male rhesus macaques. Psychological Science. DOI: :10.1177/0956797611415543

Leach, C. W., Spears, R., Branscombe, N. R., \& Doosje, B. (2003). Malicious pleasure: Schadenfreude at the suffering of another group. Journal of Personality and Social Psychology, 84, 932-943. DOI: 10.1037/0022-3514.84.5.932 
Madden, T. J., Hewitt, K., \& Roth, M. S. (2000), Managing images in different cultures: a cross-national study of color meanings and preferences. Journal of International Marketing, 8, 90-107. DOI: 10.1509/jimk.8.4.90.19795

Saito, M. (1996). A comparative study of color preferences in Japan, China and Indonesia, with emphasis on the preference for white. Perceptual and Motor Skills, 83,115-128. DOI: 10.2466/PMS.83.5.115-128

Valdez, P., \& Mehrabian, A. (1994). Effects of color on emotions. Journal of Experimental Psychology: General, 123, 394-409. DOI: 10.1037/0096-3445.123.4.394 
Color of a Nation 20

\section{Author Note}

The author would like to thank Iris Schneider, Karin Lemmens, and Anne Maass for helpful comments on a previous version of this manuscript. 


\section{Footnotes}

${ }^{1}$ Although the colors of the Dutch flag are red, white and blue, the national color is orange, referring to the first king of the Netherlands, William I, Prince of Orange. The flag is based on the Prince's Flag, which was orange, white and blue.

${ }^{2}$ Average age of the participants differed between measurement times, $F(2,217)=$ $8.11, p<.001$. Participants were slightly older during the EC $(M=22.34, S D=4.12)$, than during the post-measurement $(M=20.04, S D=2.80), t(182)=4.50, p<.001$, while participants in the pre-measurement did not differ significantly in age from participants in the other two measurements $(M=21.00, S D=5.44, p$ 's $>.10)$. Age was not related to the color evaluations or levels of national identification ( $p$ 's $>.10)$. Controlling for age in the regression analyses performed in Study 2 improved significance levels somewhat, but age was not included in the final model due to the lack of clear a-priori predictions regarding the relationship between age and evaluations of the color orange.

${ }^{3}$ On the $21^{\text {st }}$ of June, the Dutch team lost against Russia during the knock-out stage of the tournament. Regrettably, due to a programming error, data collected in the week immediately following the loss against Russia was not stored.

${ }^{4}$ Twenty people who did not participate in Study 1 to 3 were asked to name each of the colors that were used as dependent variables in Study 2 and 3. As expected, all the words used by the participants to describe the colors matched the words used to refer to the colors in the current article. Thus, there was no ambiguity in the color stimuli used in the studies.

${ }^{5}$ Both the main sponsor of the Dutch national soccer team during the last European championship (Nationale Nederlanden) as the current main sponsor (ING) have orange company logos. 